\title{
Intensity and predictability of background noise as determinants of simple reaction time
}

\author{
DAVID L. KOHFELD and DENNIS W. GOEDECKE \\ Southern Illinois University, Edwardsville, Illinois 62026
}

\begin{abstract}
This research permits a comparison of the effects of background-noise intensity (105 dB, $70 \mathrm{~dB}$, and no noise) and mode of presentation (unpredictable, predictable, and self-administered) on simple reaction time (RT) to auditory and visual test signals. Each of 112 subjects participated in a 1-h session comprised of 146 RT trials. The background noise was presented during the intertrial interval, and the average exposure duration was $2 \mathrm{sec} /$ trial. It was found that at $105-\mathrm{dB}$ noise levels, intensity was the crucial variable, whereas at $70-\mathrm{dB}$ levels, the predictability of the background noise was of primary importance. It was concluded that background noise debilitates RT performance when the noise is too loud, or, if the noise is moderately intense, because it is temporally unpredictable.
\end{abstract}

At least two simple reaction time (RT) experiments have shown that when subjects were exposed to intense stimulation just prior to participation in a RT session, mean RT was considerably longer than when other subjects were preexposed to moderate or weak stimuli (Kohfeld, 1968; Murray \& Kohfeld, 1965). In Kohfeld's experiment, subjects were seated in a sound-treated room and required to listen to 12 brief presentations of a stimulus over a period of $4 \mathrm{~min}$. Separate groups were preexposed to $35-, 65-$, or $100-\mathrm{dB}$ tones. Three additional groups of subjects were given corresponding intensities of white noise in the same manner. A warning light was presented just prior to the onset of a noise or tone in order to increase the predictability of the preexposure stimulus. Immediately following these procedures, a conventional RT task was administered. The results indicated that mean RT was fastest for the groups preexposed to the 35-dB stimuli, slowest for the groups preexposed to $100 \mathrm{~dB}$, and intermediate for the subjects exposed to the $65-\mathrm{dB}$ stimuli. Subsequent research (Kohfeld, 1969a, 1969b) has also shown that the background stimuli which influence RT performance do not have to be presented prior to the task itself, but can be distributed as ready signals throughout the entire RT session.

Recent research by Glass and Singer (1972) revealed that the predictability of background noise has a major effect on postnoise task performance. They also divided their experimental session into two phases: a noise-

This research was supported by Research Grant 020457 from the Office of Research and Projects, Southern Illinois University, Edwardsville, to D. L. Kohfeld, and is based on a Master's thesis submitted to Southern Illinois University, Edwardsville, by D. W. Goedecke. We are indebted to Robert Ruhl for the instrumentation involved in the experiment. Requests for reprints should be sent to David L. Kohfeld, Department of Psychology, Southern Illinois University, Edwardsville, Illinois 62026 . exposure session and a postnoise performance session. The two dimensions of noise were its intensity and predictability. Predictable noise was given at regular intervals throughout the exposure session, while unpredictable noise was presented randomly with respect to the onset and duration of each noise burst. It was found that unpredictable background noise had a pronounced effect on postnoise task performance. The subjects who were preexposed to either 108- or 56-dB unpredictable noise were the least persistent in their attempts to trace insoluble puzzles; the subjects exposed either to strong or weak predictable noise were equally persistent in their problem-solving attempts, and did not differ from a no-noise group. These results indicated that the predictability of background noise was an important determinant of postnoise performance, whereas noise intensity was relatively inconsequential. Furthermore, another experiment revealed that when subjects were given perceived control over the 108-dB unpredictable noise during the exposure, they performed just as well on postnoise tasks as subjects who received no noise. Based on these results, Glass and Singer concluded that the perception of having no control, and the concomitant stress of this perception, is the intervening variable relating unpredictable background noise to the poor quality of postnoise performance.

The results of Glass and Singer's (1972) research raise the interesting possibility that the predictability (and/or controlability) of background stimuli could also be a relevant variable in the RT paradigm. Accordingly, our present experiment was designed to evaluate the possible effects of the intensity, predictability, and controlability of background noise on RT performance. The experiment involved a four-way design comprising three levels of Variable A (unpredictable, predictable, and self-administered background noise), two levels of 
Variable B (105- and 70-dB background noise), two levels of Variable $\mathrm{C}$ (white-light and white-noise response signals), and three levels of Variable D (60-, 70-, and 80-dB response-signal intensities). A no-noise control group was also included in the study. Variables $\mathrm{A}$ and $\mathrm{B}$ are related to what we term a predictability hypothesis and an intensity hypothesis, respectively. If a strict version of a predictability hypothesis is confirmed, a main effect due to Variable A should result (RT would be slowest in the unpredictable-noise conditions), while the effect of Variable B should be insignificant. On the other hand, if a strict intensity hypothesis holds, Variable B should produce a significant effect (RT would be slowest in the 105-dB-noise conditions), and Variable A should have no main effect. Of course, if an A by B interaction were obtained, a more subtle analysis of the two hypotheses would be appropriate.

\section{METHOD}

\section{Subjects}

The subjects were 56 male and 56 female undergraduates enrolled at Southern Illinois University, Edwardsville. Participation in the experiment was one way to fulfill a research requirement in a sophomore-level introductory psychology class. Sixteen subjects, eight males and eight females, were randomly assigned to each of the seven background-noise groups, which consisted of either 105- or 70-dB predictable, unpredictable, or self-administered background noise, or the $0-\mathrm{dB}$ (no-noise) control condition. All subjects had normal or corrected vision and normal hearing.

\section{Apparatus}

The experiment was conducted in a sound-treated chamber which was totally dark throughout the entire experimental session. The subjects sat in a desk chair and responded to the signals by tapping a telegraph key as quickly as possible to the onset of the response signals. The auditory signals were three white-noise signals of $1.5-\mathrm{sec}$ duration, having intensities of 60,70 , and $80 \mathrm{~dB}$, re SPL $\left(20 \mathrm{microN} / \mathrm{m}^{2}\right)$ for a $1,000-\mathrm{Hz}$ tone. All noise levels were produced by a white-noise generator $(0-20,000 \mathrm{~Hz})$ and, after appropriate attenuation, were presented through calibrated earphones by means of an electronic switch with a rise and decay time of $25 \mathrm{msec}$. The visual signals (1.5-sec duration) were three corresponding levels of white fluorescent light at intensities of 60,70 , and $80 \mathrm{~dB}$, re $10^{-10} \mathrm{~L}$ $\left(.31827 \mathrm{microcd} / \mathrm{m}^{2}\right)$. These levels of light and sound have previously been shown to generate equivalent RT-intensity functions (Kohfeld, 1971). The light levels were produced by three fluorescent tubes whose outputs were regulated by dimming circuits for each tube. A dark-adapted observer calibrated these levels with a Macbeth illuminometer. The lights were presented through a $381 \times 381 \mathrm{~mm}$ window of $3.18-\mathrm{mm}$ milky Plexiglas. The window was situated in front of a light box, the center of which was mounted at eye level approximately $.91 \mathrm{~m}$ from the subject.

A tactual ready signal was employed in order to control for possible cross-modal effects of the ready signal on the visual and auditory RT-intensity functions (Kohfeld, 1969a). The ready signal was administered by a .5-sec contact with three small buttons installed on the arm of a silent relay that was attached underneath a card table upon which the subject's left forearm was resting. Actuation of the relay caused the buttons to move upward slightly and touch the subject's left index and middle fingers. The ready signal was followed by a foreperiod interval of 1,2 , or $3 \mathrm{sec}$, randomly assigned to trials. Timing of events was regulated by interval timers operating in a repetitive sequence. Two paper-tape readers and two shielded relay trees were used to program the three foreperiod intervals, the six RT signals, and the appropriate combination of background noise during the intertrial interval (ITI). RT was registered in milliseconds on each trial by a digital read-out counter.

\section{Procedure}

Figure 1 represents a temporal display of the seven possible ways to present the noise on a given trial in the unpredictablebackground-noise conditions. The background-noise stimuli were administered during the 16-sec ITI, which is illustrated across the top of Figure 1. The seven possible combinations of noise are listed in the left panel. In the body of Figure 1, one single dot represents one 1-sec noise burst, two dots joined by a solid line indicate one 2-sec noise burst, and three connected dots represent one 3-sec noise burst. Thus, for example, a subject might receive one 1-sec noise burst during the 9 th $\mathrm{sec}$ of the ITI on trial $\mathrm{n}$ (row 1 in Figure 1) and perhaps one 1-sec burst during the 5 th sec, followed by a 2-sec burst during the 10th and 11 th sec on trial $n+1$ (row 4), depending on the trial-to-trial sequence. The order of the seven possible presentations of unpredictable background noise was randomized across trials, with the restriction that the average length of ITI noise in an entire session was $2 \mathrm{sec} /$ trial. There was a $3-\mathrm{sec}$ buffer time from response-signal offset to the first second in which it was possible to get a noise burst (row 3), and a 4-sec buffer time between the last second that it was possible to get a noise burst and the onset of the ready signal (rows 3 and 6 in Figure 1).

In the predictable-background-noise conditions, one 2-sec noise burst, as shown in row 5 of Figure 1, was delivered on every trial. A $10-\mathrm{W}$ light was illuminated briefly $2 \mathrm{sec}$ prior to the onset of the predictable noise burst. In the self-administeredbackground-noise conditions, the subject depressed a foot switch to initiate the onset of the one $2-\mathrm{sec}$ noise burst. The foot switch had to be depressed before the 12 th sec of the ITI, or the 2 -sec noise burst was presented automatically at that time. Thus, it was possible for the subject to control the time of onset of the noise, but it was not possible to avoid the 2-sec noise burst.

Each subject participated in a single 1-h session consisting of 146 scored trials. The subject was given conventional RT instructions and additional information concerning the intensity and predictability of the background noise that would be presented. The subjects in the self-administered-background-noise conditions were instructed concerning the use of the foot switch, which would initiate the burst of noise during the ITI. They were also told that if they chose not to depress the switch, the noise would be delivered automatically. These instructions

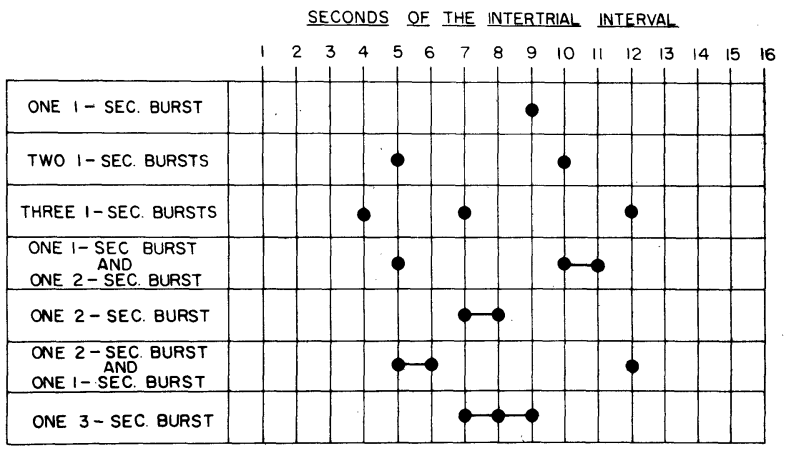

Figure 1. Temporal distributions of the noise bursts of the seven combinations of unpredictable background noise. 
were followed by a 5-min period of partial dark adaptation, during which the subject sat quietly in the darkened sound booth. The subjects were then instructed over an intercom to adjust their earphones, place the first and second fingers of their left hand on the ready-signal buttons, and place their right index finger on the telegraph key. The subjects were asked to keep their eyes open and look straight ahead during all of the RT trials. Each subject received 15 unscored practice trials to insure that all of the instructions were completely understood. The experimental session was divided into two blocks of 73 trials each, with a brief rest period between the blocks. One block of trials consisted of a random presentation of the three white-light signals, whereas the three white-noise signals were presented randomly in the other block. The order of presentation of the two blocks was counterbalanced across subjects, and each signal intensity was presented equally of ten within each block of trials. All subjects in each of the seven background-noise conditions received the same random sequence of response signals.

\section{RESULTS}

In accordance with the design outlined previously, the mean RT for each of the response signals was calculated for each subject and entered into the computation of a 3 by 2 by 2 by 3 [type of background noise (A) by intensity of background noise (B) by signal modality (C) by signal intensity (D)] mixed-design analysis of variance. First, this analysis revealed no significant differences in mean RT to comparable levels of light and noise signals (Variable C) $[\mathrm{F}(1,135)=1.52]$. Moreover, Variable $\mathrm{C}$ did not interact significantly with any of the other three variables in the analysis. Accordingly, mean RTs were collapsed over modalities for clearer graphic presentation.

The left panel of Figure 2 indicates that the no-noise control condition produced shorter RTs than either the 105- or 70- $\mathrm{dB}$ unpredictable-background-noise conditions. The apparent difference between the RT functions of the latter two conditions was not statistically significant $[t(30)=.91]$. The center panel shows that the 105-dB predictable-background-noise group produced slower RTs than either the 70-dB predictable-backgroundnoise condition or the no-noise control, the data for the latter two conditions being almost identical. Finally, the right panel of Figure 2 reveals that the three selfadministered-background-noise conditions were similar to their corresponding predictable-background-noise conditions, a conclusion confirmed by a series of simple test comparisons. It is also obvious upon close examination of all three panels in Figure 2 that RT performance was equivalent for the three 105-dB background-noise conditions.

In regard to the major hypotheses outlined previously, neither the between-subjects effect of Variable A (type of background noise) nor the between-subjects effect of Variable $B$ (intensity of background noise) reached statistical significance $[F(2,90)=2.29, p>.10$, and $F(1,90)=2.26, p>.10$, respectively]. The withinsubjects effect of Variable D (signal intensity) was, as

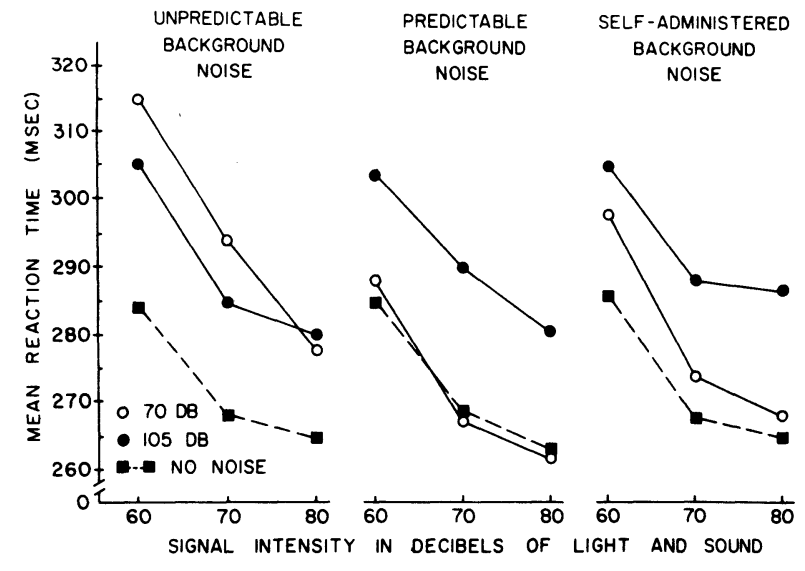

Figure 2. Mean reaction time as a function of the signal intensities, collapsed across white light and white noise, for the three modes of presentation of background-noise intensity. (The signal intensities are specified in decibels of white light, re $.31827 \mathrm{microcd} / \mathrm{m}^{2}$, and white noise, re $20 \mathrm{microN} / \mathrm{m}^{2}$ for $1,000-\mathrm{Hz}$ tone.)

usual, very large $[F(2,180)=352.53, p<.001]$. The fact that Variables A and B were not significant becomes more reasonable in view of two considerations. First, the A by $B$ interaction was significant $[F(2,90)=3.45$, $\mathrm{p}<.05]$. In other words, when the 70- and 105-dB noise levels were evaluated in the four-way analysis of variance, neither the predictability hypothesis nor the intensity hypothesis could account for the data until we examined how they interacted. This is apparent when one compares the 70-dB background-noise groups across the three panels of Figure 2. Clearly, the 70-dB unpredictable-background-noise condition produced slower RTs than either the $70-\mathrm{dB}$ predictable- or the 70-dB self-administered-background-noise conditions. This observation received support from a two-way analysis of variance which compared the three $70-\mathrm{dB}$ background-noise conditions as a function of the signal intensities $[F(2,45)=8.90, p<.001]$. Second, although the results from the no-noise control group were included in the study, it was inappropriate to enter the data from this group into the four-way analysis (three control groups would have been necessary to satisfy statistical requirements, one for each level of Variable A). Alternatively, three simple tests which included the control group permitted separate comparisons of the effects of 105-, 70-, and 0-dB levels in each of the three modes of background noise. The appropriate tests were intensity of background noise (3) by signal intensity (3) analyses of variance, computed separately for the unpredictable, predictable, and self-administered background-noise conditions. In all three cases the intensity of background noise was significant $[F(2,45)=$ $4.66, \mathrm{p}<.05 ; \mathrm{F}(2,45)=4.08, \mathrm{p}<.05 ;$ and $\mathrm{F}(2,45)=$ $4.74, p<.05$, respectively]. Since the control group performed in zero or near-zero background noise, the results of the analyses that included this group support 
the intensity hypothesis, but do not refute the predictability hypothesis, in view of the A by B interaction obtained in the major four-way analysis of variance.

Another group of simple tests in the self-administeredbackground-noise condition deserves mention. It may be recalled that the subjects in the two self-administeredbackground-noise conditions had the option of choosing when, during the intertrial interval, they "wished" to listen to the background noise. Given this option, the time between the presentation of the background noise and the presentation of the response signal could vary between 4.5 and $17.5 \mathrm{sec}$, depending upon whether the subject was a "late pusher" (one who waited at least $6 \mathrm{sec}$ before initiating the noise burst) or an "early pusher" (one who initiated the noise burst $4 \mathrm{sec}$ or less after the start of the intertrial interval). Simple tests comparing the performance of early pushers with that of late pushers for both the 105- and 70-dB selfadministered-background-noise conditions revealed nonsignificant results $[\mathrm{t}(10)=.33$ and $\mathrm{t}(11)=.78$, respectively]. These tests indicate that differences in the time between presentation of the self-administered background noise and presentation of the response signal did not influence subsequent RT performance.

\section{DISCUSSION}

The main reason for this research was to determine the conditions in which background noise debilitates simple task performance. Our analyses indicate that if the noise is loud enough, its sheer intensity interferes with RT performance. However, at moderate levels of background noise its predictability becomes an important factor. In this vein, our results show that, if moderate noise is unpredictable, RT is just as slow as when the noise is very intense. On the other hand, if the moderate noise is predictable, whether signaled or selfadministered, RT is equally rapid as in no-noise conditions. An implication of these data is that somewhere between 70 and $105 \mathrm{~dB}$ there exists a level of background noise above which no degree of cognitive control (i.e., predictability) can change its debilitating effect on simple RT performance. Conversely, there exists another level of background noise below which the predictability and/or controlability of its intensity could be helpful to a person engaged in simple task performance.

Another important outcome of our experiment was that the effects of background noise were not restricted to the processing of auditory signals in the RT task. Not only was there no signifi- cant difference in overall mean RT to light and noise signals, but also the effect of the background-noise conditions was relatively equal for the visual and auditory response signals. This finding indicates that the effects of background noise are not restricted to peripheral sensory mechanisms, but reflect processes that are more central in nature. Indeed, we think that if the present study was replicated using background light, similar results would be obtained. These interpretations are consistent with the data obtained by Kohfeld (1969a), in which equally intense auditory and visual ready signals produced the same results in a RT task with auditory response signals.

Two aspects of Glass and Singer's (1972) research deserve comment in relation to our present data. First, they utilized proofreading and problem-solving tasks which presumably did not require very rapid signal detection, while we used a RT task which required the rapid detection of sensory information. It is possible that the predictability of background noise is the relevant variable in problem-solving situations, whereas both the predictability and the intensity of noise are potentially important in tasks that require rapid signal detection. Second, their view that the adverse effects of unpredictable noise are based on the loss of perceived control was evaluated further in our self-administered-noise conditions. At moderate (70-dB) background-noise levels, our results are consistent with Glass and Singer's, in that both predictability and controlability proved beneficial to RT performance. Whether the predictability or the perceived control of noise is the relevant construct seems less important than the fact that both variables produced similar effects in problem-solving and signal-detection tasks.

\section{REFERENCES}

Glass, D. C., \& Singer, J. E. Behavioral aftereffects of unpredictable and uncontrollable aversive events. American Scientist, 1972, 60, 457-465.

KoHFELD, D. L. Stimulus intensity and adaptation level as determinants of simple reaction time. Journal of Experimental Psychology, 1968, 76, 468-473.

KoHFELD, D. L. Effects of the intensity of auditory and visual ready signals on simple reaction time. Journal of Experimental Psychology, 1969, 82, 88-95. (a)

KoHFELD, D. L. Effects of ready-signal intensity. and intensity of the preceding response signal on simple reaction time. American Journal of Psychology, 1969, 82, 104-110. (b)

KohfELD, D. L. Simple reaction time as a function of stimulus intensity in decibels of light and sound. Journal of Experimental Psychology, 1971, 88, 251-257.

Murray, H. G., \& Kohfeld, D. L. Role of adaptation level in stimulus intensity dynamism. Psychonomic Science, 1965, 3, 439-440.

(Received for publication April 24, 1978.) 